Pensamiento Crítico Vol. $18 \mathrm{~N}^{\circ} 2$, pp. 43-60

\title{
Análisis comparativo del desarrollo de los sistemas bancarios del Perú y Chile \\ Gaby Cortez Cortez
}

\section{RESUMEN}

El grado de desarrollo y de profundidad del sistema bancario del Perú se encuentra por debajo del nivel promedio de América Latina durante los años 2006 a 2012, mientras que el de los bancos de Chile se ubica por encima de dicho promedio. En cuanto a la concentración, los bancos de Chile están menos concentrados que los bancos del Perú, lo que nos mostraría el mayor poder de mercado que tienen los bancos del Perú. De manera general, la rentabilidad de los bancos del Perú es superior a la de los bancos de Chile. De otro lado, en cuanto a la estructura del crédito los bancos de Chile se diferencian porque adicionalmente a financiar prioritariamente a las empresas, el crédito al sector hipotecario es el segundo en importancia, mientras que en los bancos del Perú, los recursos financieros se dirigen en primer lugar a las empresas y en segundo lugar al crédito de consumo.

Palabras clave: Sistema bancario, desarrollo.

\section{ABSTRACT}

The degree of development and depth of Peru's banking system is below the average for Latin America during the years 2006-2012, while the banks of Chile is above that average. In terms of concentration, Chilean banks are less concentrated 


\section{Pensamiento Crítico Vol. 18. $\mathrm{N}^{\circ} 2$}

than the banks of Peru, which would show the increased market power of the Peruvian banks. Generally the profitability of banks in Peru is higher than the banks in Chile. On the other hand, in terms of the credit structure Chilean banks differ primarily because in addition to finance companies, the credit to mortgage industry is second in importance, while in the case of Peruvian banks, the financial resources target at companies first and second to consumer credit.

Keywords: Banking, system, development .

\section{INTRODUCCIÓN}

El desarrollo del sistema financiero y en especial el del sistema bancario es uno de los elementos importantes en la determinación del grado de competitividad de los países, asimismo nos proporciona una aproximación del acceso a los servicios financieros por parte de las familias y las empresas; por lo que considero interesante llevar a cabo un análisis del desenvolvimiento comparativo del sistema bancario del Perú y de un país próximo como Chile.

\section{INDICADORES GENERALES}

\section{Profundidad Bancaria}

Una manera de medir el grado de profundidad de un sistema bancario es a través de la penetración bancaria, que puede ser calculada mediante el Crédito / PBI y/o los Depósitos / PBI. Es decir que cuanto mayor es este indicador, mayor será el nivel de profundización bancaria de un país. Se observa que la penetración del crédito es de 77.8 por ciento para Chile y de 27.2 por ciento para el Perú en el 2012, situándose el primer país por encima del promedio para América Latina, mientras que el Perú se encuentra por debajo del promedio. Ver Gráfico 1. 


\section{Gaby Cortez Cortez}

GRÁFICO N 1. Crédito / PBI del Sistema Bancario de América Latina: Nov. 2012(\%).

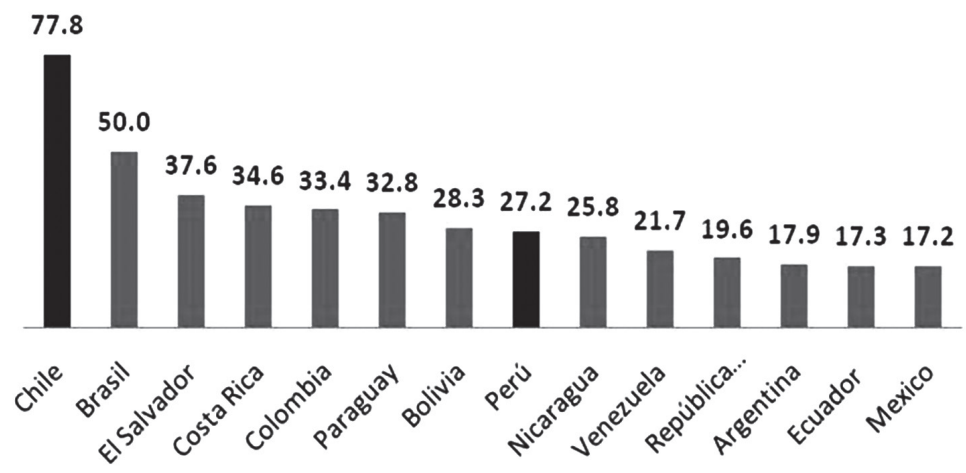

De otro lado, la penetración de los depósitos es de 67.3 por ciento para Chile, mientras que para el Perú es de 26.5 por ciento, ubicándose en el primer caso por encima del promedio de América Latina y en el caso del Perú por debajo de dicho promedio. Ver el Gráfico 2. Estos hechos nos muestran que el grado de penetración de los bancos en el Perú es menor que el de los bancos de Chile, y similar al resto de países de América Latina. Estos indicadores nos muestran que el desarrollo del sistema bancario del Perú debe mejorar a fin de incorporar a más segmentos del público no atendido.

GRÁFICO Nº 2. Depósitos / PBI del Sistema Bancario de América Latina: Nov. 2012(\%).

\section{3}

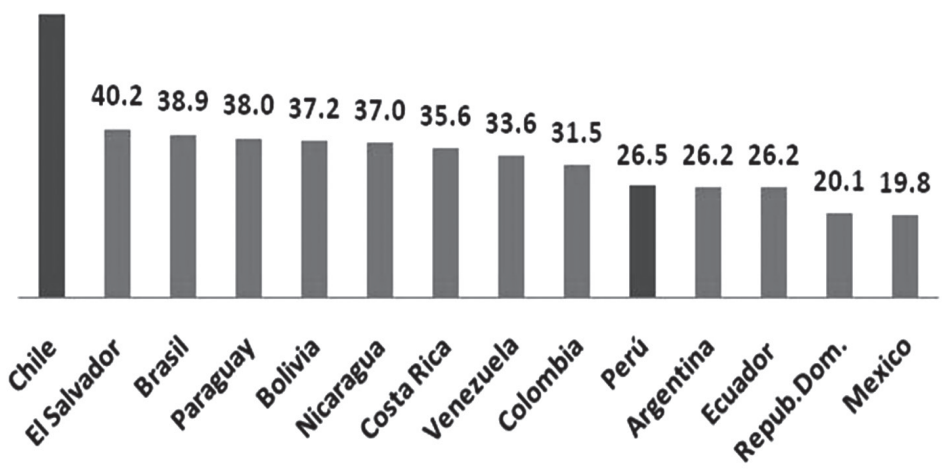




\section{Calidad de la Cartera de Activos}

Una manera de medir la gestión de los bancos es a través de la calidad de la cartera de colocaciones mediante los Préstamos con problemas / Préstamos totales, observándose que para la mayor parte de los países de América Latina este indicador promedio ha venido disminuyendo del 2006 al 2012.

Para el caso de los bancos de Chile, durante los años 2006 al 2008 este ratio se situaba muy por debajo del promedio de los bancos de América del Sur, pero se incrementa del 2009 hasta el 2011, situándose ligeramente por encima del promedio señalado, lo que nos indica el manejo cuidadoso de los malos préstamos. Ver el CUADRO 1 y Gráfico 3.

En el caso de los bancos del Perú, se produce una disminución de este ratio desde 4.1 por ciento en el 2006 hasta 2.75 en el 2012, y de manera similar a los bancos de Chile se ubica ligeramente sobre el promedio de América Latina en los tres últimos años. Parece que se aprendió la lección de la crisis de la banca de 1997 y 1998, cuando el Estado tuvo que comprar la cartera pesada de los bancos privados, para evitar una crisis en el sistema bancario.

CUADRO N ${ }^{\circ}$. Préstamos con problemas / Préstamos totales de Bancos de América Latina (\%): 2006-2012.

\begin{tabular}{lcccccccc}
\hline & 2006 & 2007 & 2008 & 2009 & 2010 & 2011 & 2012 & Prom. \\
\hline Argentina & 3.4 & 2.7 & 2.7 & 3.0 & 1.8 & 1.2 & 1.5 & 2.3 \\
Bolivia & 8.7 & 5.6 & 4.3 & 3.5 & 2.2 & 1.7 & 1.47 & 3.92 \\
Brazil & 3.5 & 3.0 & 3.1 & 4.2 & 3.1 & 3.5 & 3.45 & 3.41 \\
Chile & $\mathbf{0 . 8}$ & $\mathbf{0 . 8}$ & $\mathbf{1 . 0}$ & $\mathbf{2 . 9}$ & $\mathbf{2 . 7}$ & $\mathbf{2 . 4}$ & $\mathbf{2 . 1 6}$ & $\mathbf{1 . 8 2}$ \\
Colombia & 2.7 & 3.2 & 3.9 & 4.0 & 2.9 & 2.5 & 2.76 & 3.14 \\
Ecuador & 4.1 & 3.7 & 3.4 & 4.1 & 3.5 & 3.2 & & 3.7 \\
Mexico & 1.8 & 2.4 & 3.0 & 2.8 & 2.0 & 2.1 & 2.40 & 2.36 \\
Paraguay * & 3.3 & 1.3 & 1.1 & 1.6 & 1.3 & 1.7 & 2.30 & 1.80 \\
Perú & $\mathbf{4 . 1}$ & $\mathbf{2 . 7}$ & $\mathbf{2 . 2}$ & $\mathbf{2 . 7}$ & $\mathbf{3 . 0}$ & $\mathbf{2 . 9}$ & 2.75 & 2.91 \\
Uruguay & 3.7 & 1.1 & 1.0 & 1.2 & 1.0 & 1.3 & 1.50 & 1.54 \\
Venezuela & 1.1 & 1.2 & 1.9 & 3.0 & 3.4 & 1.4 & 0.88 & 1.84 \\
\hline Promedio & $\mathbf{3 . 4}$ & $\mathbf{2 . 5}$ & $\mathbf{2 . 5}$ & $\mathbf{3 . 0}$ & $\mathbf{2 . 4}$ & $\mathbf{2 . 2}$ & 2.1 & \\
\hline FMl & * A julio 2012 & & & & & &
\end{tabular}




\section{Gaby Cortez Cortez}

GRÁFICO N 3. Préstamos con Problemas / Préstamos Totales de Bancos en América Latina: 2006-2012 (\%).

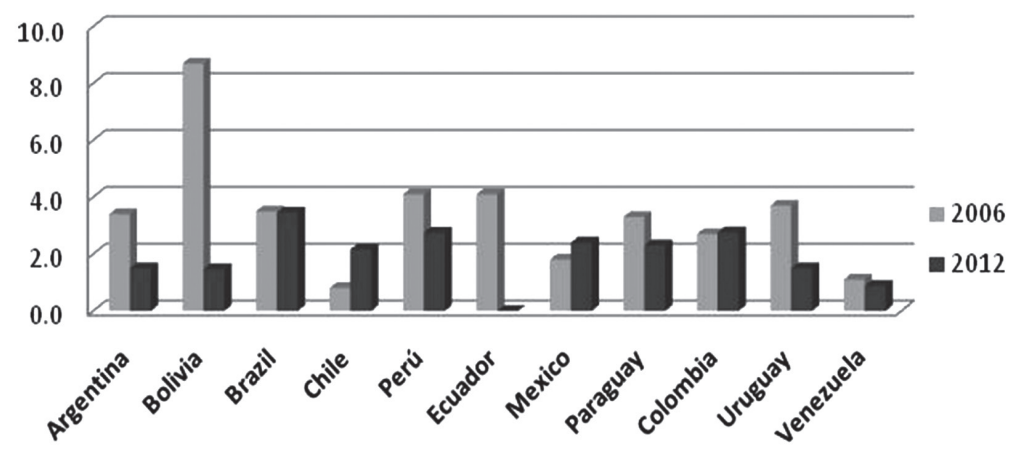

Una de las formas con la que los bancos buscan enfrentar los riesgos es a través de la conformación de Provisiones / Préstamos en problemas. De esta forma los bancos de América Latina han incrementado la proporción de estas provisiones desde 141.9 por ciento en promedio en el 2006, hasta 164.6 por ciento en el 2012. Ver el CUADRO 2 y el Gráfico 4.

En el caso de Chile, los bancos han disminuido las provisiones desde 198.2 por ciento en el 2006 hasta 98.5 por ciento en el 2011, sin embargo debe mencionarse que del 2006 al 2008 tales porcentajes estuvieron por encima del promedio de los bancos de América Latina. Este comportamiento nos indica que la percepción de riesgo ha disminuido durante estos años, incluso durante la crisis. En el caso del Perú, la constitución de provisiones se incrementa desde 100.3 por ciento en el 2006 hasta 142.5 por ciento en el 2012, y se mantiene bastante cerca del promedio de la región, en algunos años. Como se sabe, las provisiones constituyen un costo para los bancos, el que luego será trasladado a los prestatarios, encareciendo el costo final de los préstamos para los clientes, y de alguna manera mide también la eficiencia de los créditos otorgados, es decir, la calidad de la gerencia de los bancos. 


\section{Pensamiento Crítico Vol. 18. $\mathrm{N}^{\circ} 2$}

CUADRO N ${ }^{\circ}$ 2. Provisiones bancarias / Préstamos en problemas de Bancos de América Latina: 2006-2012 (\%).

\begin{tabular}{lcccccccc}
\hline & 2006 & 2007 & 2008 & 2009 & 2010 & 2011 & 2012 & Prom. \\
\hline Argentina & 108.2 & 115.2 & 117.2 & 115.3 & 147.7 & 160.0 & 143.7 & 129.6 \\
Bolivia* & 106.5 & 132.4 & 153.7 & 165.7 & 214.1 & 228.2 & 266.9 & 181.1 \\
Brazil & 179.9 & 181.9 & 189.0 & 157.3 & 171.1 & 167.1 & 152.0 & 171.2 \\
Chile & $\mathbf{1 9 8 . 2}$ & $\mathbf{2 0 9 . 8}$ & $\mathbf{1 7 9 . 0}$ & $\mathbf{8 1 . 1}$ & $\mathbf{9 2 . 7}$ & $\mathbf{9 8 . 5}$ & $\mathbf{1 0 3 . 2}$ & $\mathbf{1 3 7 . 5}$ \\
Colombia & 154.4 & 133.8 & 124.3 & 140.1 & 174.0 & 168.7 & 164.0 & 151.3 \\
Ecuador & 161.7 & 170.0 & 168.2 & 162.3 & 171.3 & 170.0 & - & 167.3 \\
Mexico & 210.3 & 168.9 & 161.2 & 173.9 & 200.7 & 181.5 & 185.0 & 183.1 \\
Paraguay** & 59.1 & 78.2 & 77.7 & 78.8 & 74.4 & 66.7 & 63.1 & 71.1 \\
Perú & $\mathbf{1 0 0 . 3}$ & $\mathbf{1 3 1 . 6}$ & $\mathbf{1 5 1 . 0}$ & $\mathbf{1 3 9 . 3}$ & $\mathbf{1 4 1 . 9}$ & $\mathbf{1 4 6 . 0}$ & $\mathbf{1 4 2 . 5}$ & $\mathbf{1 3 6 . 1}$ \\
Uruguay & 52.8 & 55.1 & 63.1 & 72.8 & 72.2 & 70.1 & 73.0 & 65.6 \\
Venezuela & 229.1 & 175.7 & 148.0 & 136.4 & 149.1 & 202.7 & 352.7 & 199.1 \\
\hline Promedio & $\mathbf{1 4 1 . 9}$ & $\mathbf{1 4 1 . 1}$ & $\mathbf{1 3 9 . 3}$ & $\mathbf{1 2 9 . 4}$ & $\mathbf{1 4 6 . 3}$ & $\mathbf{1 5 0 . 9}$ & $\mathbf{1 6 4 . 6}$ & \\
\hline FMI & & * A marzo 2012 & $* *$ A julio & 2012 & & &
\end{tabular}

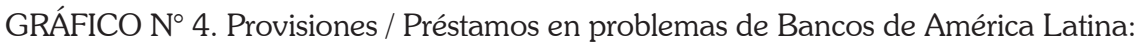
2006-2012 (\%).

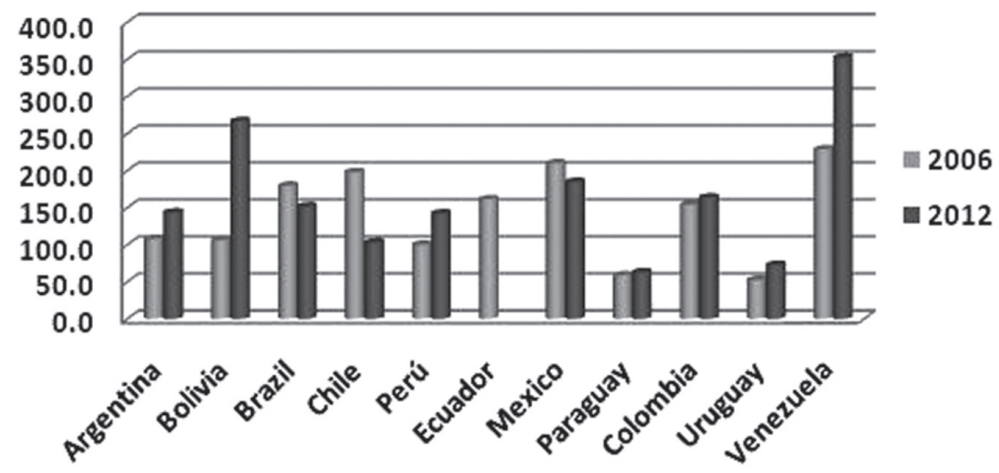




\section{Gaby Cortez Cortez}

\section{Rentabilidad Bancaria}

Una manera de medir la rentabilidad de las empresas es a través de la Utilidad / Capital o también conocido como ROE por sus siglas en Inglés Return On Equity. El ratio de rentabilidad promedio para América Latina ha disminuido levemente desde 24.4 en el 2006 hasta 23.6 en el 2012. Ver el CUADRO 3 y el Gráfico 5.

Para el caso de Chile el ROE ha pasado de 22.5 por ciento en el 2006 a 17.3 por ciento en el 2012, experimentando en términos generales una disminución durante estos años; asimismo es necesario mencionar que durante los años 2006 al 2008 el ROE de los bancos de Chile se situó por debajo del promedio de la región, mientras que del 2009 al 2011 el ROE fue ligeramente superior al promedio de América Latina.

De otro lado los bancos del Perú han tenido un comportamiento del ROE que parte de 22.7 por ciento en el 2006 y llega a 22.4 por ciento en el 2012 con un nivel superior al de los bancos de Chile durante todos estos años. En este sentido, debe señalarse que durante el 2007 y 2008 el ROE de los bancos del Perú alcanzaron niveles de 28.3 y 31.3 por ciento respectivamente; situándose muy por encima del promedio de la región en estos años y en los siguientes.

CUADRO N³. Utilidad / Capital (ROE) de los Bancos de América Latina: 2006-2012 (\%).

\begin{tabular}{lcccccccc}
\hline & 2006 & 2007 & 2008 & 2009 & 2010 & 2011 & 2012 & Prom. \\
\hline Argentina & 14.3 & 11.0 & 13.4 & 19.2 & 24.3 & 23.3 & 25.8 & 18.8 \\
Bolivia & 8.6 & 21.2 & 20.3 & 20.6 & 17.3 & 16.9 & 17.6 & 17.5 \\
Brazil & 29.0 & 31.3 & 14.3 & 22.1 & 29.3 & 29.6 & 15.9 & 24.5 \\
Chile & $\mathbf{2 2 . 5}$ & $\mathbf{1 8 . 6}$ & $\mathbf{1 8 . 5}$ & $\mathbf{2 1 . 4}$ & $\mathbf{2 0 . 7}$ & $\mathbf{2 4 . 6}$ & $\mathbf{1 7 . 3}$ & $\mathbf{2 0 . 5}$ \\
Colombia & 29.9 & 30.8 & 28.1 & 26.2 & 27.2 & 18.7 & 21.2 & 26.0 \\
Ecuador & 17.9 & 15.3 & 12.1 & 10.3 & 11.3 & 15.3 & - & 13.7 \\
Mexico & 31.2 & 24.1 & 14.8 & 15.2 & 16.8 & 16.0 & 17.5 & 19.4 \\
Paraguay* & 41.7 & 34.2 & 38.0 & 30.9 & 30.5 & 29.1 & 27.6 & 33.1 \\
Perú & $\mathbf{2 2 . 7}$ & $\mathbf{2 8 . 3}$ & $\mathbf{3 1 . 3}$ & $\mathbf{2 4 . 5}$ & $\mathbf{2 4 . 2}$ & $\mathbf{2 4 . 3}$ & $\mathbf{2 2 . 4}$ & $\mathbf{2 5 . 4}$ \\
Uruguay & 19.0 & 18.0 & 16.3 & 5.5 & 12.1 & 11.8 & 17.5 & 14.3 \\
Venezuela & 31.6 & 32.4 & 29.4 & 16.9 & 20.0 & 33.9 & 53.0 & 31.0 \\
\hline Promedio & $\mathbf{2 4 . 4}$ & $\mathbf{2 4 . 1}$ & $\mathbf{2 1 . 5}$ & $\mathbf{1 9 . 3}$ & $\mathbf{2 1 . 2}$ & $\mathbf{2 2 . 1}$ & $\mathbf{2 3 . 6}$ & \\
\hline FMI & * Julio & 2012 & & & & & &
\end{tabular}




\section{Pensamiento Crítico Vol. 18. $\mathrm{N}^{\circ} 2$}

GRÁFICO N 5. Utilidad / Capital (ROE) de los Bancos de América Latina: 2006-2012 (\%).

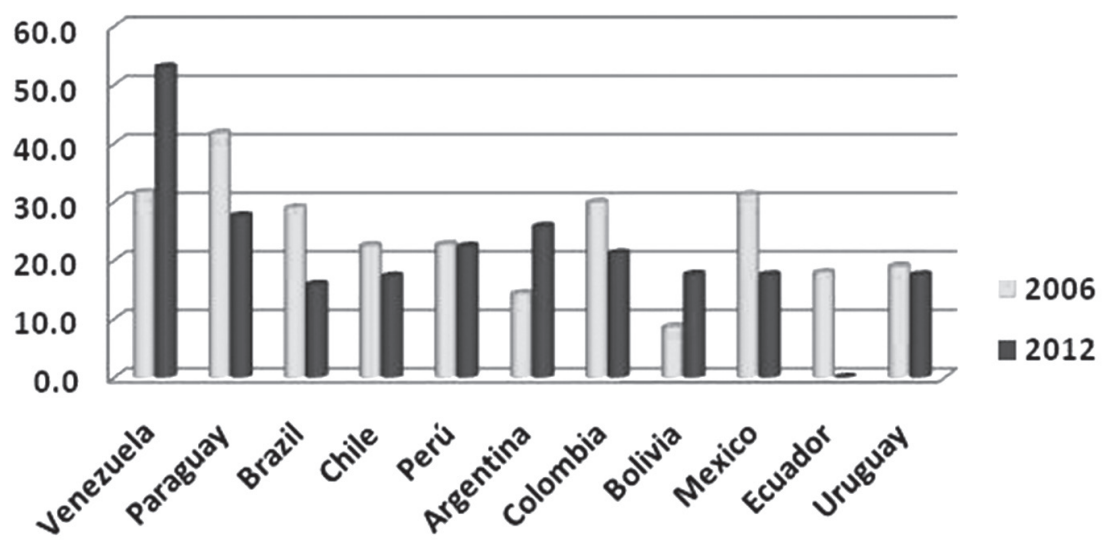

Otro indicador importante de la rentabilidad de los bancos es el que se obtiene de dividir la Utilidad / Activos Totales, o también conocido como ROA o Return on Assets, el que nos indica que tan bien se han usado los activos en la obtención de cierto nivel de utilidad.

El ROA promedio de la región fue de 2.5 en el 2006 y de 2.3 en el 2012, indicando una ligera disminución especialmente del 2007 al 2009. Ver CUADRO 4 y Gráfico 6.

Los bancos de Chile tuvieron un ROA por debajo del promedio de América Latina del 2006 al 2012, sin embargo debe notarse que del 2009 al 2011 se produce una ligera recuperación en este ratio. Asimismo, debe mencionar que el ROA de los bancos de Chile ha sido inferior al de los bancos del Perú durante todos estos años.

Los bancos del Perú por otro lado, tuvieron un ROA por encima del promedio de la región con excepción del 2006. 


\section{Gaby Cortez Cortez}

CUADRO N 4 . Utilidad / Activos de los Bancos de América Latina: 2006-2012 (\%).

\begin{tabular}{lcccccccc}
\hline & 2006 & 2007 & 2008 & 2009 & 2010 & 2011 & 2012 & Prom. \\
\hline Argentina & 1.9 & 1.5 & 1.6 & 2.3 & 2.8 & 2.5 & 2.9 & 2.2 \\
Bolivia & 0.9 & 1.9 & 1.7 & 1.7 & 1.4 & 1.3 & 1.4 & 1.5 \\
Brazil & 3.1 & 3.4 & 1.5 & 2.4 & 3.2 & 3.3 & 1.6 & 2.6 \\
Chile & $\mathbf{1 . 5}$ & $\mathbf{1 . 3}$ & $\mathbf{1 . 2}$ & $\mathbf{1 . 5}$ & $\mathbf{1 . 7}$ & $\mathbf{1 . 9}$ & $\mathbf{1 . 4}$ & $\mathbf{1 . 5}$ \\
Colombia & 4.0 & 4.0 & 3.6 & 3.5 & 3.9 & 2.7 & 3.1 & 3.5 \\
Ecuador & 2.3 & 2.1 & 1.7 & 1.5 & 1.6 & 2.1 & - & 1.9 \\
Mexico & 2.8 & 2.3 & 1.4 & 1.5 & 1.8 & 1.6 & 1.8 & 1.9 \\
Paraguay* & 4.4 & 3.4 & 3.9 & 2.9 & 2.8 & 2.9 & 2.6 & 3.3 \\
Perú & $\mathbf{2 . 1}$ & $\mathbf{2 . 5}$ & $\mathbf{2 . 6}$ & $\mathbf{2 . 3}$ & $\mathbf{2 . 4}$ & $\mathbf{2 . 3}$ & $\mathbf{2 . 2}$ & $\mathbf{2 . 3}$ \\
Uruguay & 1.7 & 1.8 & 1.6 & 0.5 & 1.2 & 1.2 & 1.5 & 1.4 \\
Venezuela & 3.0 & 2.6 & 2.5 & 1.5 & 1.8 & 2.9 & 4.1 & 2.6 \\
\hline Promedio & $\mathbf{2 . 5}$ & $\mathbf{2 . 4}$ & $\mathbf{2 . 1}$ & $\mathbf{2 . 0}$ & $\mathbf{2 . 2}$ & $\mathbf{2 . 2}$ & $\mathbf{2 . 3}$ & \\
\hline FMI & $* 2012$ a julio & & & & & &
\end{tabular}

GRÁFICO Nº 6. Utilidad / Activos (ROA) de los Bancos de América Latina: 2006-2012 (\%).

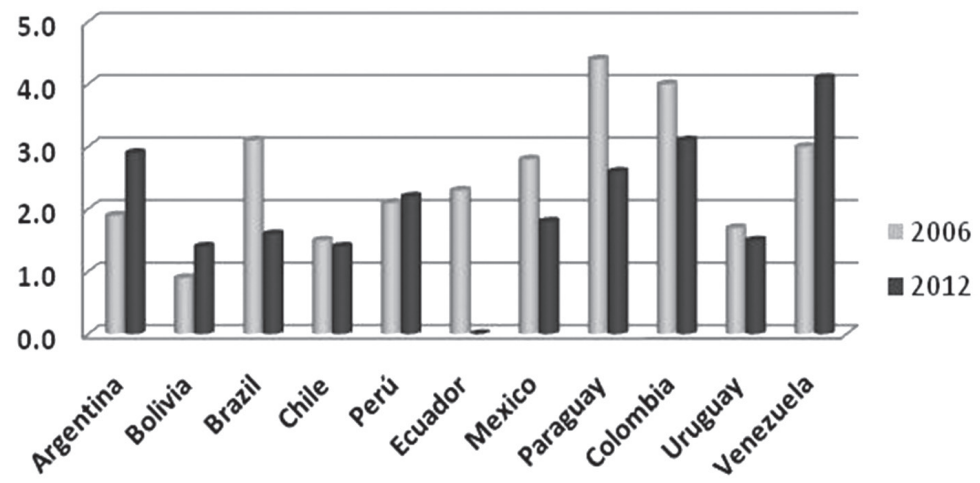

\section{Capital Bancario}

Los bancos son empresas que tienen una característica especial, son empresas con un grado de apalancamiento bastante alto, es decir son financiados con recursos 
del público fundamentalmente. En tal sentido, el capital de estas instituciones es un elemento importante para la salud financiera de las mismas, así como para la protección frente a los riesgos en los que incurren estas empresas. Los bancos como cualquier empresa se enfrentan a riesgos a fin de conseguir una mayor rentabilidad, para lo cual deben incrementar su capital.

Una manera de medir este desenvolvimiento es a través del ratio de Capital Bancario / Activos. El ratio promedio para América Latina, ha tenido un ligero aumento al pasar de 9.9 por ciento en el 2006 a 10.3 por ciento en el 2012. Ver el CUADRO 5 y el GRÁFICO 7.

Los bancos de Chile tuvieron un ratio de capital / activos por debajo del promedio de la región del 2006 al 2011. Un comportamiento similar experimentaron los bancos del Perú al situarse dicho indicador por debajo del promedio de la región, pero a un nivel más alto que el de los bancos chilenos.

CUADRO N 5. Capital Bancario / Activos de los Bancos en América Latina: 2006-2012 (\%).

\begin{tabular}{lcccccccc}
\hline & 2006 & 2007 & 2008 & 2009 & 2010 & 2011 & 2012 & Prom. \\
\hline Argentina & 13.6 & 13.7 & 12.9 & 13.3 & 11.9 & 11.2 & 11.8 & 12.6 \\
Bolivia & 10.0 & 9.0 & 9.3 & 8.7 & 8.4 & 8.5 & 8.6 & 8.9 \\
Brazil & 10.8 & 11.3 & 10.7 & 11.4 & 11.1 & 11.3 & 10.1 & 11.0 \\
Chile & $\mathbf{6 . 8}$ & $\mathbf{7 . 1}$ & $\mathbf{6 . 4}$ & $\mathbf{6 . 9}$ & $\mathbf{8 . 3}$ & $\mathbf{7 . 6}$ & $\mathbf{8 . 0}$ & $\mathbf{7 . 3}$ \\
Colombia & 13.3 & 12.9 & 12.6 & 14.2 & 14.3 & 14.4 & 14.7 & 13.8 \\
Ecuador & 8.2 & 8.1 & 8.9 & 9.4 & 8.9 & 8.8 & - & 8.7 \\
Mexico & 9.5 & 9.6 & 9.2 & 10.7 & 10.4 & 10.1 & 10.6 & 10.0 \\
Paraguay* & 8.5 & 9.7 & 10.2 & 9.5 & 9.4 & 10.1 & 10.1 & 9.6 \\
Perú & $\mathbf{9 . 5}$ & $\mathbf{8 . 8}$ & $\mathbf{8 . 3}$ & $\mathbf{1 0 . 2}$ & $\mathbf{9 . 5}$ & $\mathbf{9 . 3}$ & $\mathbf{1 0 . 0}$ & $\mathbf{9 . 4}$ \\
Uruguay & 9.4 & 10.5 & 8.9 & 9.8 & 9.5 & 10.8 & 8.3 & 9.6 \\
Venezuela & 9.8 & 9.4 & 9.4 & 8.6 & 9.9 & 10.2 & 10.4 & 9.7 \\
\hline Promedio & $\mathbf{9 . 9}$ & $\mathbf{1 0 . 0}$ & $\mathbf{9 . 7}$ & $\mathbf{1 0 . 2}$ & $\mathbf{1 0 . 1}$ & $\mathbf{1 0 . 2}$ & $\mathbf{1 0 . 3}$ & \\
\hline FMI & & $*$ A julio & $\mathbf{2 0 1 2}$ & & & & &
\end{tabular}




\section{Gaby Cortez Cortez}

GRÁFICO N 7. Capital / Activo de Bancos de América Latina (\%): 2006-2012.

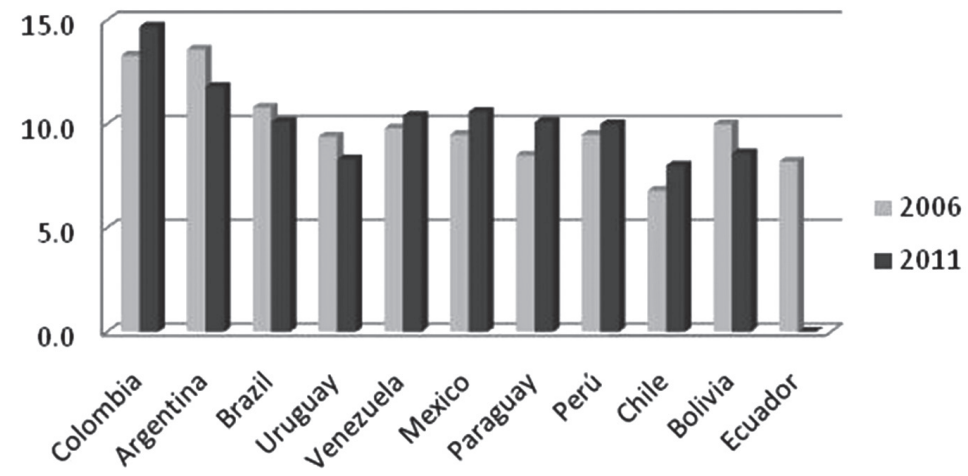

Continuando con esta reflexión, una manera más apropiada de medir el capital necesario frente a los riesgos en que incurren los bancos es usando el ratio del Capital Regulatorio / Activos Ponderados por Riesgo.

Es así que se observa que para América Latina el ratio promedio de capital regulatorio ha mostrado una ligera disminución que va desde 15.7 por ciento en el 2006 a 14.9 por ciento en el 2012. En el intermedio de estos años, después de la crisis financiera del 2008 se incrementa este ratio en el 2009, para luego descender nuevamente. Ver el CUADRO 6 y el GRÁFICO 8.

Al igual que en el caso anterior, los bancos de Chile tuvieron un capital regulatorio por debajo del promedio indicando de esta manera que están incurriendo en riesgos esperados.

Los bancos del Perú también tienen un ratio de capital regulatorio por debajo del de los bancos de América Latina, pero a nivel menor que el de los bancos de Chile, con excepción del 2012. 


\section{Pensamiento Crítico Vol. 18. № 2}

CUADRO N 6. Capital Regulatorio / Activos Ponderardo por Riesgo de Bancos de América Latina: 2006-2012.

\begin{tabular}{lcccccccc}
\hline & 2006 & 2007 & 2008 & 2009 & 2010 & 2011 & 2012 & Prom. \\
\hline Argentina & 16.9 & 16.9 & 16.9 & 18.8 & 17.7 & 16.5 & 17.1 & 17.3 \\
Bolivia & 13.3 & 12.6 & 13.7 & 13.3 & 11.9 & 12.0 & 10.1 & 12.4 \\
Brazil & 18.9 & 18.7 & 18.2 & 18.9 & 17.6 & 18.2 & 16.1 & 18.1 \\
Chile & 12.5 & 12.2 & $\mathbf{1 2 . 5}$ & $\mathbf{1 4 . 3}$ & $\mathbf{1 4 . 1}$ & $\mathbf{1 3 . 6}$ & $\mathbf{1 3 . 3}$ & $\mathbf{1 3 . 2}$ \\
Colombia & 15.4 & 16.0 & 15.4 & 17.2 & 17.3 & 17.4 & 18.1 & 16.7 \\
Ecuador & & 19.2 & 19.9 & 19.6 & 18.3 & 18.2 & & 19.0 \\
Mexico & 16.1 & 15.9 & 15.3 & 16.5 & 16.9 & 16.5 & 16.0 & 16.2 \\
Paraguay* & 20.1 & 16.8 & 16.3 & 16.3 & 16.4 & 16.9 & 16.8 & 17.1 \\
Perú** & 12.5 & 11.7 & $\mathbf{1 1 . 9}$ & $\mathbf{1 3 . 5}$ & $\mathbf{1 3 . 7}$ & $\mathbf{1 3 . 2}$ & $\mathbf{1 4 . 6}$ & $\mathbf{1 3 . 0}$ \\
Uruguay & 16.9 & 17.8 & 16.7 & 16.8 & 15.2 & 17.1 & 12.8 & 16.2 \\
Venezuela & 14.3 & 12.9 & 13.4 & 14.0 & 13.2 & 12.6 & 14.3 & 13.5 \\
\hline Promedio & $\mathbf{1 5 . 7}$ & $\mathbf{1 5 . 5}$ & $\mathbf{1 5 . 5}$ & $\mathbf{1 6 . 3}$ & $\mathbf{1 5 . 7}$ & $\mathbf{1 5 . 7}$ & $\mathbf{1 4 . 9}$ & \\
\hline FMI & *A julio 2012 & & & &
\end{tabular}

GRÁFICO N 8. Capital Regulatorio / Activos Ponderardo por Riesgo de Bancos: 2006-2012 (\%).

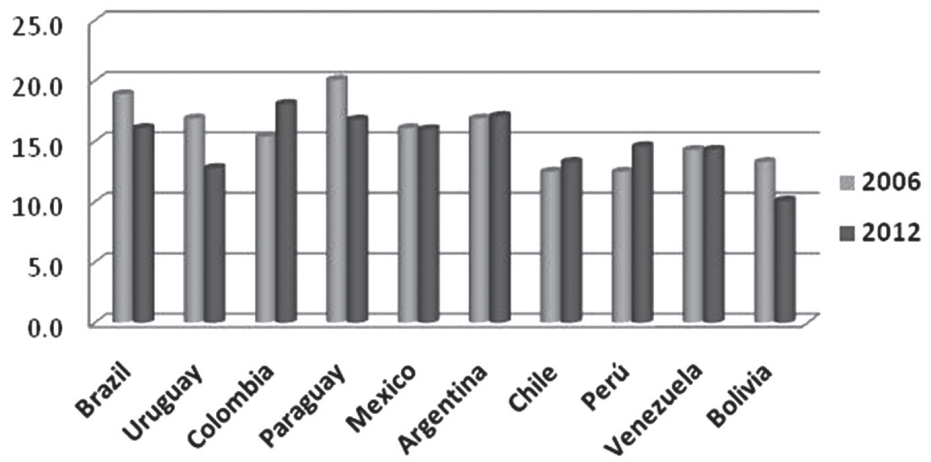

\section{ANÁLISIS DEL CRÉDITO BANCARIO DE CHILE}

El crédito del sistema bancario de Chile se ha incrementado del 2008 al 2012 en cada año, pasando de US\$109,664 MM en el 2008 a US\$205,715 MM, observándose la mayor tasa de crecimiento en el año 2012 (24.9 por ciento) y ha tenido un crecimiento acumulado de 87.6 por ciento del 2008 al 2011. Ver el Gráfico 9. 


\section{Gaby Cortez Cortez}

GRÁFICO N 9. Crédito del Sistema Bancario de Chile 2008-2012 (MMUS\$).

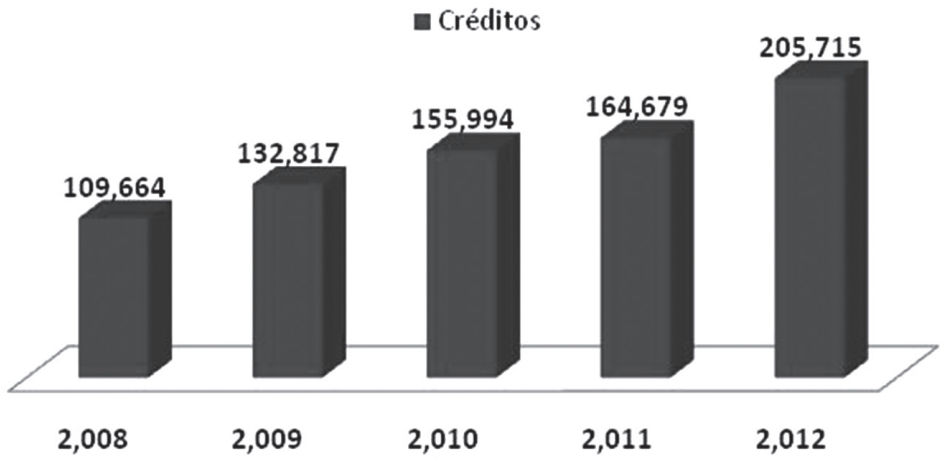

Se puede observar que el sistema bancario de Chile concentra el 81.14 por ciento de las colocaciones en los seis primero bancos, indicándonos que es un sistema menos concentrado que el de otros países de América Latina. Ver el CUADRO 7.

CUADRO N 7. Banca de Chile: Participación en Colocaciones (\%).

\begin{tabular}{lcccc}
\hline Bancos & 2009 & 2010 & 2011 & 2012 \\
\hline De Chile & 19.3 & 19.4 & 19.9 & 18.7 \\
Santander Chile & 19.5 & 20.7 & 19.6 & 18.6 \\
Del Estado Chile & 15.8 & 15.2 & 14.4 & 13.9 \\
De Crédito e Inversiones & 12.7 & 12.7 & 12.8 & 12.9 \\
Corpbanca & $\mathbf{7 . 2}$ & $\mathbf{7 . 3}$ & $\mathbf{7 . 8}$ & 10.1 \\
BBVA de Chile & $\mathbf{7 . 3}$ & $\mathbf{7 . 3}$ & $\mathbf{7 . 0}$ & $\mathbf{7 . 0}$ \\
Scotiabank Chile & 6.0 & 5.3 & 5.0 & 4.9 \\
Itaú Chile & 3.2 & 3.4 & 3.9 & 4.2 \\
Security & 3.1 & 2.6 & 2.9 & 3.0 \\
Bice & 2.7 & 2.6 & 2.8 & 2.8 \\
Falabella & 0.9 & 1.0 & 1.1 & 1.1 \\
Internacional & 0.6 & 0.8 & 0.8 & 0.7 \\
Rabobank Chile & 0.4 & 0.5 & 0.6 & 0.7 \\
HSBC Bank Chile & 0.4 & 0.4 & 0.4 & 0.3 \\
Consorcio & 0.0 & 0.2 & 0.3 & 0.4 \\
Ripley & 0.3 & 0.3 & 0.2 & 0.2 \\
París & 0.3 & 0.2 & 0.2 & 0.2 \\
Otros (*) & 0.4 & 0.3 & 0.4 & 0.5 \\
\hline Total & 100.0 & 100.0 & 100.0 & 100.0 \\
\hline Fuente: SBIF de Chile & Elaboración: Gaby Cortez & & \\
\hline & & & &
\end{tabular}




\section{Pensamiento Crítico Vol. 18. $\mathrm{N}^{\circ} 2$}

De otro lado, la estructura del crédito de los bancos de Chile por grandes sectores, nos muestra que en un 64 por ciento en promedio estos recursos se dirigieron a las empresas, el 25 por ciento a vivienda y el 13 por ciento se destinó al consumo. Ver el CUADRO 8.

CUADRO No 8. Sistema Bancario de Chile: Créditos por Sectores 2008-2012. MM US\$ y porcentajes.

\begin{tabular}{rccccc}
\hline & Empresas & Consumo & Vivienda & Provision & Total Crédito \\
\hline 2008 & 66 & 12 & 24 & -2 & 109,664 \\
2009 & 63 & 13 & 26 & -2 & 132,817 \\
2010 & 62 & 13 & 27 & -3 & 155,994 \\
2011 & 63 & 13 & 26 & -2 & 164,679 \\
2012 & 64 & 14 & 25 & -2 & 205,715 \\
\hline Promedio & 64 & 13 & 25 & -2 & 153,774 \\
\hline
\end{tabular}

Fuente: SBIF de Chile

Elaboración: Gaby Cortez

Se ha tomado un grupo de once bancos del total de bancos de Chile para analizar su comportamiento respecto al destino de sus colocaciones y se encuentra que en un 59 por ciento dirigen estos recursos a las empresas, en un 24.2 por ciento a vivienda y en un 15.7 por ciento al consumo, al mes de marzo del 2013. Ver el CUADRO 9.

Hay bancos como Bice por ejemplo que dirige el 84.4 por ciento de sus recursos a las empresas, mientras que Security destina el 76.7 por ciento, Corpbanca y BCI consignan el 73.0 y 67.7 respectivamente. El banco Itaú de Chile y el De Chile, dirigen el 66.0 y el 61.4 por ciento cada uno. Estos bancos destinan la mayor parte de sus recursos a las empresas y se sitúan por encima del promedio de los bancos.

De otro lado, los bancos que destinan una proporción importante de sus recursos a la vivienda son Santander, del Estado, BBVA Chile, Scotiabank y Falabella. 


\section{Gaby Cortez Cortez}

CUADRO N 9. Colocaciones de Bancos de Chile por Sectores (\%).

\begin{tabular}{|c|c|c|c|}
\hline \multirow[b]{3}{*}{ Bice } & \multicolumn{3}{|c|}{2013 Marzo } \\
\hline & Empresas & Consumo & Vivienda \\
\hline & 84.4 & 3.1 & 10.9 \\
\hline Security & 76.7 & 6.9 & 16.3 \\
\hline Corpbanca & 73.0 & 10.8 & 15.3 \\
\hline $\mathrm{BCl}$ & 67.7 & 12.6 & 19.1 \\
\hline Itaú Chile & 66.0 & 13.2 & 20.8 \\
\hline De Chile & 61.4 & 14.8 & 22.2 \\
\hline Santander & 55.2 & 16.5 & 27.6 \\
\hline Estado & 53.7 & 9.1 & 34.4 \\
\hline BBVA & 52.2 & 15.1 & 31.9 \\
\hline Scotiabank & 52.1 & 9.6 & 36.5 \\
\hline Falabella & 7.5 & 61.3 & 31.2 \\
\hline Promedio & 59.1 & 15.7 & 24.2 \\
\hline
\end{tabular}

\section{CRÉDITO DEL SISTEMA BANCARIO PERUANO}

En el CUADRO 10 se puede observar la estructura del crédito del sistema bancario peruano durante el periodo 2005 a marzo 2013, en donde se aprecia que en promedio durante estos años, el 68 por ciento de estos recursos se dirigieron a las empresas (Crédito corporativo, Grande, Mediana, Pequeña y Microempresa); el 17 por ciento al consumo y el 14 por ciento a crédito hipotecario.

Es aquí en donde encontramos la primera diferencia con la estructura del sistema bancario de Chile, en donde el crédito de consumo fue de 13 por ciento en promedio y de 25 por ciento para vivienda, manteniendo la mayor proporción para el crédito a las empresas.

En el caso de los bancos del Perú se observa una tendencia a la disminución dentro de la estructura de recursos que se dirigen a las empresas a favor de los recursos que se destinan al consumo especialmente, ya que el crédito a las empresas pasa de participar del 71 por ciento del crédito total en el 2005 a 65 por ciento a marzo 2013. Ver el GRÁFICO 10. 


\section{Pensamiento Crítico Vol. 18. $\mathrm{N}^{\circ} 2$}

Un aspecto importante a destacar es que el número de deudores de la banca se incrementa desde 2, 350,965 en el 2005 a 6, 013,083 a marzo del 2013.

CUADRO N 10. Sistema Bancario del Perú: Créditos Directos por sectores 2005-2013*.

\begin{tabular}{cccccc}
\hline \multicolumn{2}{c}{ Empresas ** } & Consumo & Hipotecario & Total Crédito & Núm.Deudores \\
\hline 2005 & 71 & 14 & 15 & $43,676,262$ & $2,350,965$ \\
2006 & 70 & 16 & 14 & $50,338,757$ & $2,853,178$ \\
2007 & 69 & 18 & 12 & $66,829,333$ & $3,925,840$ \\
2008 & 69 & 18 & 12 & $91,879,144$ & $5,100,384$ \\
2009 & 69 & 18 & 14 & $92,397,309$ & $4,298,314$ \\
2010 & 69 & 17 & 14 & $109,721,572$ & $4,805,823$ \\
2011 & 67 & 18 & 15 & $128,377,902$ & $5,274,856$ \\
2012 & 65 & 18 & 16 & $144,165,311$ & $5,845,699$ \\
2013 & 65 & 18 & 17 & $157,978,287$ & $6,013,083$ \\
\hline Promedic & 68 & 17 & 14 & $98,373,764$ & $4,496,460$ \\
\hline
\end{tabular}

Fuente: SBS Perú

Elaboración: Gaby Cortez

* A Julio

**Crédito Corporativo, Grande, Mediana, Pequeña y Microempresa

GRÁFICO N 10. Crédito Bancario Directo en el Perú por sectores: 2013.

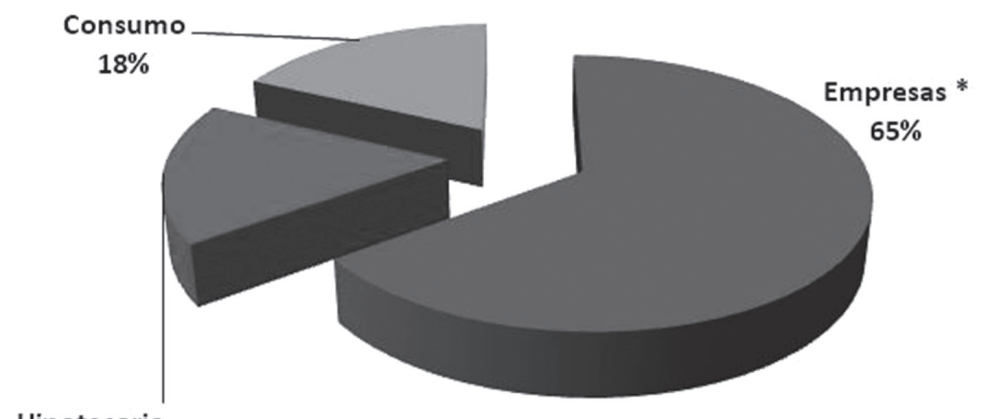

Hipotecario

$17 \%$ 


\section{Gaby Cortez Cortez}

\section{CONCLUSIONES Y RECOMENDACIONES}

El grado de profundidad del sistema bancario (Crédito/PBI y Depósitos/PBI) en el Perú se encuentra por debajo del nivel del promedio de América Latina, mientras que el de Chile se ubica por encima de dicho promedio.

La calidad de la cartera de colocaciones de los bancos (Préstamos en problemas/ Total de préstamos) del Perú y de Chile se ha mejorado en los últimos años, situándose alrededor del promedio del 2009 al 2011 en ambos casos.

El nivel de provisiones para los bancos de Chile ha disminuido especialmente del 2009 al 2011, situándose por debajo del promedio de América Latina, mientras que los bancos del Perú mantienen sus provisiones alrededor del nivel promedio de la región, indicándonos la mayor toma de riesgo y a su vez el encarecimiento de los recursos financieros.

En términos generales la rentabilidad (ROE) de los bancos de Chile se situó por debajo del promedio de la región del 2006 al 2008 y del 2009 y 2011, alrededor del promedio; mientras que el ROE de los bancos del Perú se situó por encima del promedio, indicándonos la mayor rentabilidad de los banco domésticos.

Durante los años 2006 al 2011 El ROA de los bancos de Chile se ubico por debajo del promedio de América Latina, mientras que el ROE de los bancos del Perú, estuvo por encima del promedio, indicándonos nuevamente la mayor rentabilidad de los bancos que operan en el Perú.

El capital bancario y el capital regulatorio de Chile y Perú se encuentran por debajo del nivel promedio de América Latina señalándonos que el nivel de riesgo en

que incurren los bancos de ambos países está siendo manejado de manera adecuada, a la luz de la experiencia de la crisis de los 98-99 para el caso peruano.

Los bancos de Chile se encuentran menos concentrados ya que seis bancos dan cuenta del 81.14 por ciento de las colocaciones totales a diciembre de 2012, mientras que en el caso de los bancos del Perú son cuatro bancos los que concentran el 83.25 por ciento. A mayor concentración, mayor poder de mercado y facilidad para imponer condiciones a los clientes. 


\section{Pensamiento Crítico Vol. 18. $\mathrm{N}^{\circ} 2$}

La estructura de las colocaciones de los bancos de Chile a diciembre 2012 y de años anteriores nos muestra que el 64 por ciento de estos créditos fueron a las empresas, el 14 por ciento al consumo y el 25 por ciento a vivienda; mientras que las provisiones constituían el -3 por ciento.

En el caso de los bancos del Perú, el 65 por ciento de los créditos se destina a las empresas, el 18 por ciento al consumo y el 16 a vivienda. Se aprecia la mayor participación de los créditos al consumo en el caso del Perú y de los créditos de vivienda en el caso de Chile. Esto nos indicaría que de alguna manera se estaría desarrollando un mercado de crédito hipotecario en el sistema bancario de Chile en base a cierta estabilidad y a la posible negociación de los productos financieros resultantes. En el caso de los bancos del Perú, se privilegia el consumo mediante el incremento importante de tarjetas de crédito, pero que son ofertadas con cautela por los bancos grandes y con mayor dinamismo por los bancos medianos y por algunos bancos-almacén pequeños.

\section{RECOMENDACIÓN}

Considero que es necesario profundizar en el estudio comparativo de los sistemas bancarios del Perú y de Chile, así como con otros países de la región, a fin de poder contrastar mejor el estado de desarrollo de nuestro sistema financiero, que es una herramienta importante en la competitividad global, así como en el otorgamiento de financiamiento a las empresas y familias.

\section{REFERENCIAS BIBLIOGRÁFICAS}

Memorias del Banco Central de Reserva del Perú. www.bcrp.gob.pe

Estadísticas del Banco Central de Reserva del Perú. www.bcrp.gob.pe

Estadísticas de la Superintendencia de Banca, Seguros y AFPs del Perú. www.sbs.gob.pe

Superintendencia de Bancos e Instituciones Financieras de Chile. www.sbif.cl

Memorias del Banco Central de Reserva de Chile. www.bcentral.cl

BBVA Research Group. www.research.com

Chile IMF Country Report 2013. www.imf.org

Financial Deepening and International Monetary Stability 2011. www.imf.org 